\title{
Evaluation of Influence of terrain profile on the physical characteristics of air flow
}

\author{
Sh.E.Guseynov ${ }^{1,2,3}$ \\ ${ }^{1}$ Faculty of Science and Engineering, Liepaja University, Liepaja, Latvia \\ ${ }^{2}$ Institute of Fundamental Science and Innovative Technologies, Liepaja, Latvia \\ 3"Entelgine" Research \& Advisory Co., Ltd., Riga, Latvia \\ E-mails: sh.e.guseinov@inbox.lv
}

\begin{abstract}
In the present paper, a measure of the influence of irregularities of the underlying surface (for instance, the real terrain profile) on the dynamics of air flow is estimated. It is shown that these irregularities significantly perturbing the pressure, density, velocity and temperature of air flow; for perturbed pressure, density, velocity and temperature of air flow the closed formulas in the Fourier integral form are obtained. In addition, in the present paper, it is proved that due to these perturbations, at a certain altitude of the atmosphere some surface of the "sturdy wall" form appears, on which the vertical and horizontal components of the air flow velocity become infinitesimal and infinitely large, respectively.
\end{abstract}

Keywords: mathematical model; terrain profile; air flow fluctuations; asymptotic expansion Mathematics Subject Classification: 76G25, 35E99, 34B15, 00A71

\section{Introduction}

As it is known (for instance, see [1-4]), roughness of Earth surface affect the way bodies are streamlined by the air flow or liquid. Ignoring Earth terrain when building mathematical models of pollutants moving in the air and on aquatic environment do not allow to distinguish arising perturbations of air flow, namely, in various scientific papers (see [5-7]), which are dedicated to the research of pollutants dynamics in the atmosphere/liquid, the corresponding mathematical models are constructed within the canonical domains (1D - a rod of certain or infinite length; 2D - a rectangle or a bar; 3D - a confined or boundless parallelepiped). Thus in such models is ignored the presence of Earth surface topographic roughness.

In this work the following 2D problem is considered: assume there are two air layers $S 1$ and $S 2$, which are divided by a surface $\partial S$, and also it is supposed that $S 1$ and $S 2$ are moving parallel to the Earth surface, i.e. parallel to the horizontal surface with different velocities, $\vartheta_{1}$ and $\vartheta_{2}$. As it was already mentioned above, on the Earth surface there cannot be no arising air (or liquid) flow 
perturbations, and our aim in this work is to distinguish between the unperturbed state (which layers $S 1$ and $S 2$ would have if there were not any roughness of surface) and the perturbed state (which is true in reality). To reach this aim lets at first make such presumptions in relation to the unperturbed state of air layers $S 1$ and $S 2$ :

- characteristic measures of air layers, such as temperature, pressure, density, etc. are changing vertically, i.e. theses characteristic values depend only on the height (the reference point is the Earth surface).

- velocities $\vartheta_{1}$ and $\vartheta_{2}$ of air layers $S 1$ and $S 2$ are constant, and $\vartheta_{1} \not \equiv \vartheta_{2}$;

- temperature $T_{1}$ of the flow $S 1$ is changing linearly (see $[2-3,5]$, that is $T_{1}\left(x_{3}\right) \stackrel{\text { def }}{\equiv} T_{\text {Land }}+\alpha_{1} \cdot x_{3}$, where $T_{\text {Land }}$ is the temperature at the Earth surface; $\alpha_{1}<0$ is the temperature gradient; $x_{3}$ is the variable for the height from the Earth surface;

- temperature $T_{2}$ of the flow $S 2$ is also changing linearly $T_{2}\left(x_{3}\right) \stackrel{\text { def }}{\equiv} T_{h}+\alpha_{2} \cdot x_{3}$,

where $T_{h}$ is the temperature of the surface $\partial S$, which divides air layers $S 1$ and $S 2 ; \alpha_{2}<0$ is the temperature gradient; $h$ is the height of $\partial S$ from the Earth (as these presumptions refer to non-perturbed state, it is possible to assume that $h$ is the height of unperturbed surface of the section); $x_{3}$ is a variable height from the Earth surface;

- movement of air flows $S 1$ and $S 2$ is the same in all vertical layers, which are parallel to the flow $S 1$.

\section{Mathematical formulation of the problem}

The abovementioned problem can be introduced in such way (Fig. 1).

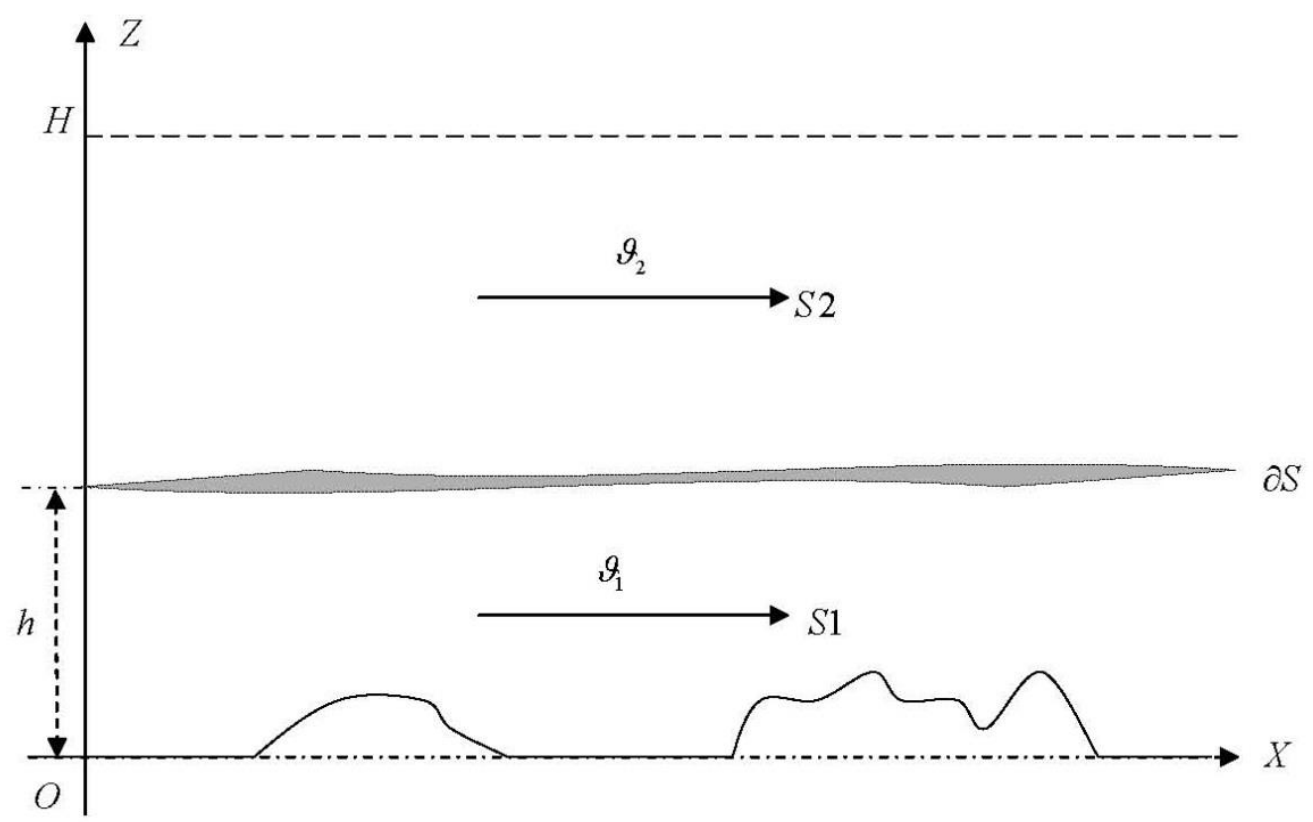




\section{Fig. 1. Movement of the airflows $S 1$ and $S 2$ with due account for Earth topography}

In order to construct a mathematical model of the problem described above, lets define $p_{1}\left(x_{3}\right)$ and $p_{2}\left(x_{3}\right)$ as pressures of air flows $S 1$ and $S 2 ; \rho_{1}\left(x_{3}\right)$ and $\rho_{2}\left(x_{3}\right)$ as densities of air flows $S 1$ and $S 2 ; \vartheta_{1}\left(x_{3}\right)$ and $\vartheta_{2}\left(x_{3}\right)$ as the velocities of air flows $S 1$ and $S 2$. As it was assumed, the temperature of air flows in $S 1$ and $S 2$ are specified by $T_{1}\left(x_{3}\right)$ and $T_{2}\left(x_{3}\right)$. Let us mention once more that $p_{i}, \rho_{i}, \vartheta_{i}$ and $T_{i}(i=1,2)$ describe amounts of air layers of unperturbed flows $S 1$ and $S 2$, but our aim is to define perturbations of the air flow arising from roughness of the Earth surface. Therefore, together with designations $p_{i}, \rho_{i}$ and $\vartheta_{i}(i=1,2)$, lets also introduce $\tilde{p}_{i}, \tilde{\rho}_{i}$, $\tilde{\vartheta}_{i}$ and $w_{i}(i=1,2)$, where $\tilde{p}_{i}$ is perturbation of pressure of the $i$-th flow $S i(i=1,2) ; \tilde{\rho}_{i}$ is perturbation of density of the $i$-th flow $S i(i=1,2) ; \tilde{\vartheta}_{i}$ is perturbation of "horizontal velocity" of the $i$-th flow $S i(i=1,2) ; w_{i}$ is perturbation of "vertical velocity" of the $i$-th air mass $S i(i=1,2)$. Then the true (real) values of such characteristic measures will obviously be equal to $p_{i}+\tilde{p}_{i}$, $\rho_{i}+\tilde{\rho}_{i}, \vartheta_{i}+\tilde{\vartheta}_{i}$ and $w_{i}(i=1,2)$, and we can write $([1-2,5-6])$ the corresponding equations of motion for these true characteristic measures:

$$
\begin{aligned}
& \frac{1}{\rho_{i}+\tilde{\rho}_{i}} \cdot \frac{\partial\left(p_{i}+\tilde{p}_{i}\right)}{\partial x_{1}}+\left(\vartheta_{i}+\tilde{\vartheta}_{i}\right) \cdot \frac{\partial\left(\vartheta_{i}+\tilde{\vartheta}_{i}\right)}{\partial x_{1}}+w_{i} \cdot \frac{\partial\left(\vartheta_{i}+\tilde{\vartheta}_{i}\right)}{\partial x_{3}}=0 ; \quad i=1,2, \\
& \left(\vartheta_{i}+\tilde{\vartheta}_{i}\right) \cdot \frac{\partial w_{i}}{\partial x_{1}}+w_{i} \cdot \frac{\partial w_{i}}{\partial x_{3}}+\frac{1}{\rho_{i}+\tilde{\rho}_{i}} \cdot \frac{\partial\left(p_{i}+\tilde{p}_{i}\right)}{\partial x_{3}}+g=0 ; \quad i=\overline{1,2}, \\
& \frac{\partial\left\{\left(\rho_{i}+\tilde{\rho}_{i}\right) \cdot\left(\vartheta_{i}+\tilde{\vartheta}_{i}\right)\right\}}{\partial x_{1}}+\frac{\partial\left\{w_{i} \cdot\left(\rho_{i}+\tilde{\rho}_{i}\right)\right\}}{\partial x_{3}}, \quad i=\overline{1,2}
\end{aligned}
$$

where

- $0<x_{1}<L ; \quad 0<x_{3}<h$ if $i=1$

- $0<x_{1}<L ; h<x_{3}<H$ if $i=2$;

- $p_{i}=p_{i}\left(x_{3}\right), \rho_{i}=\rho_{i}\left(x_{3}\right), \vartheta_{i}=\vartheta_{i}\left(x_{3}\right), \quad \tilde{p}_{i}=\tilde{p}_{i}\left(x_{1}, x_{3}\right), \quad \tilde{\rho}_{i}=\tilde{\rho}_{i}\left(x_{1}, x_{3}\right), \tilde{\vartheta}_{i}=\tilde{\vartheta}_{i}\left(x_{1}, x_{3}\right)$ and

$$
w_{i}=w_{i}\left(x_{1}, x_{3}\right) \quad(i=1,2) .
$$

It is necessary to add heat balance equation to the system (1)-(3). As we suggested that the arising perturbations are caused only by Earth surface relief (of course, there are other influences that create perturbations of these characteristic measures of air/liquid flow; refer to [1]), then heat 
balance has to be equal to zero. Hence, to the equations (1)-(3) it is necessary to add a condition of heat inflow absence, namely,

$$
\left(\vartheta_{i}+\tilde{\vartheta}_{i}\right) \cdot \frac{\partial}{\partial x_{1}}\left(\frac{p_{i}+\tilde{p}_{i}}{\left(\rho_{i}+\tilde{\rho}_{i}\right)^{\beta}}\right)+w_{i} \cdot \frac{\partial}{\partial x_{3}}\left(\frac{p_{i}+\tilde{p}_{i}}{\left(\rho_{i}+\tilde{\rho}_{i}\right)^{\beta}}\right)=0,
$$

where $\beta=1.41$.

Therefore, characteristic measures of perturbed air flows are defined (having corresponding boundary conditions and consistence conditions on the dividing surface $\partial S$ ) by a system of equations (1)-(4). In this work is studied a question of finding asymptotic expression of system (1)-(4) solution with such assumptions - perturbations of flows are so small that it is possible to take into account only their first orders; the Earth surface with minor roughness is taken. These two assumptions allow separating the system (1)-(4) into two parts, namely, the first system contains equations of unperturbed air flow motion, the second part of the system contains equations of perturbed motion. The idea of dividing the system (1)-(4) into the abovementioned parts is based on equalization of finite additives and infinitesimal of the first order in the system of equation (1)-(4) that allows to bring the original non-linear system (1)-(4) to the following two linear systems of equations:

- System of equations for unperturbed air layer

$$
\begin{aligned}
& \frac{1}{\rho_{i}} \cdot \frac{\partial p_{i}}{\partial x_{1}}=0 ; \quad i=1,2, \\
& \frac{1}{\rho_{i}} \cdot \frac{\partial p_{i}}{\partial x_{3}}+g=0 ; \quad i=1,2, \\
& \vartheta_{i} \cdot \frac{\partial \rho_{i}}{\partial x_{1}}=0 ; \quad i=1,2 ;
\end{aligned}
$$

- System of equations for perturbed air layer

$$
\begin{aligned}
& \vartheta_{i} \cdot \frac{\partial \tilde{\vartheta}_{i}}{\partial x_{1}}+\frac{1}{\rho_{i}} \cdot \frac{\partial p_{i}}{\partial x_{1}}=0, \quad i=1,2, \\
& \vartheta_{i} \cdot \frac{\partial w_{i}}{\partial x_{1}}+\frac{1}{\rho_{i}} \cdot \frac{\partial \tilde{p}_{i}}{\partial x_{3}}-\frac{\tilde{\rho}_{i}}{\rho_{i}^{2}} \cdot \frac{\partial \tilde{p}_{i}}{\partial x_{3}}=0, \quad i=1,2, \\
& \vartheta_{i} \cdot \frac{\partial \tilde{\rho}_{i}}{\partial x_{1}}+\frac{\partial\left\{\rho_{i} \cdot \tilde{\vartheta}_{i}\right\}}{\partial x_{1}}+\frac{\partial\left\{\rho_{i} \cdot w_{i}\right\}}{\partial x_{3}}=0, \quad i=1,2, \\
& \vartheta_{i} \cdot \frac{\partial p_{i}}{\partial x_{1}}-\beta \cdot R \cdot T_{i} \cdot \vartheta_{i} \cdot \frac{\partial \tilde{\rho}_{i}}{\partial x_{1}}+\left\{(\beta-1) \cdot g-\beta \cdot R \cdot \alpha_{i}\right\} \cdot \rho_{i} \cdot w_{i}=0, \quad i=1,2,
\end{aligned}
$$


where $R$ is the universal gas constant; $p_{i}, T_{i}$ and $R$ are related by the known equation $p_{i}=$ Const $\cdot e^{-\frac{g}{R} \cdot \int \frac{d \zeta}{T_{i}(\zeta)}}$

Taking into account (5)-(7) in (8)-(10) having introduced new functions $U_{i} \stackrel{\text { def }}{=} \rho_{i} \cdot w_{i}$, $\tilde{V}_{i}^{d e f}=\rho_{i} \cdot \tilde{\vartheta}_{i}, \quad i=1,2$, equations of perturbed air flow will look:

$$
\left\{\begin{array}{l}
\vartheta_{i} \cdot \frac{\partial U_{i}}{\partial x_{1}}+\frac{\partial \tilde{p}_{i}}{\partial x_{3}}+g \cdot \tilde{\rho}_{i}=0 \\
\vartheta_{i} \cdot \frac{\partial \tilde{V}_{i}}{\partial x_{1}}+\frac{\partial \tilde{p}_{i}}{\partial x_{1}}=0 \\
\vartheta_{i} \cdot \frac{\partial \tilde{\rho}_{i}}{\partial x_{1}}+\frac{\partial \tilde{V}_{i}}{\partial x_{1}}+\frac{\partial U_{i}}{\partial x_{3}}=0 \\
\vartheta_{i} \cdot \frac{\partial \tilde{p}_{i}}{\partial x_{1}}-\bar{T}_{i} \cdot \vartheta_{i} \frac{\partial \tilde{\rho}_{i}}{\partial x_{1}}+d_{i} \cdot U_{i}=0
\end{array}\right.
$$

where $\bar{T}_{i}\left(x_{3}\right) \stackrel{\text { def }}{\equiv} \beta \cdot R \cdot T_{i}\left(x_{3}\right), d_{i} \stackrel{\text { def }}{\equiv}(\beta-1) \cdot g-\beta \cdot R \cdot \alpha_{i}$.

Therefore, the problem is to find asymptotic solutions of the system (12). To do this, at first let us study an auxiliary problem, which consists of finding solution of the system (12) with particular condition that the shape of the studied domain of the Earth surface is given by an equation

$$
x_{3}=X_{3}\left(x_{1}\right)=\cos \left(\gamma \cdot x_{1}\right) .
$$

In this case it is obvious the solution of the system (12) has:

$$
\left\{\begin{array}{l}
\tilde{V}_{i}\left(x_{1}, x_{3}\right)=\tilde{V}_{i}^{(1)}\left(x_{3}\right) \cdot \cos \left(\gamma \cdot x_{1}\right), \\
\tilde{p}\left(x_{1}, x_{3}\right)=\tilde{p}_{i}^{(1)}\left(x_{3}\right) \cdot \cos \left(\gamma \cdot x_{1}\right), \\
\tilde{\rho}\left(x_{1}, x_{3}\right)=\tilde{\rho}_{i}^{(1)}\left(x_{3}\right) \cdot \cos \left(\gamma \cdot x_{1}\right), \\
U\left(x_{1}, x_{3}\right)=U_{i}^{(1)}\left(x_{3}\right) \cdot \sin \left(\gamma \cdot x_{1}\right), \\
X_{3, \partial S}\left(x_{1}\right)=C \cdot \cos \left(\gamma \cdot x_{1}\right),
\end{array}\right.
$$

where $C \equiv$ const., $X_{3, \partial S}\left(x_{1}\right)$ is surface perturbations of the section $\partial S$ (also is assumed to be an infinitesimal).

Before asking for adequacy of the system (12) to functions from (14), let's try to formulate conditions on the Earth surface and of the section $\partial S$. Since on the Earth surface normal component of particle velocity, which are adjacent to the surface, is equal to zero, then 


$$
\left.\frac{\left(\vartheta_{1}+\tilde{\vartheta}_{1}\right) \cdot \frac{d X_{3}\left(x_{1}\right)}{d x_{1}}}{\sqrt{1+\left(\frac{d X_{3}\left(x_{1}\right)}{d x_{1}}\right)^{2}}}\right|_{x_{3}=X_{3}\left(x_{1}\right)}-\left.\frac{w_{1}}{\sqrt{1+\left(\frac{d X_{3}\left(x_{1}\right)}{d x_{1}}\right)^{2}}}\right|_{z=X_{3}\left(x_{1}\right)}=0
$$

then, taking into account only equal values the first order, we obtain

$$
\vartheta_{i} \cdot \frac{d X_{3}\left(x_{1}\right)}{d x_{1}}=\left.w_{i}\left(x_{1}, x_{3}\right)\right|_{x_{3}=0} .
$$

In (15) taking into account notation $U_{1}=\rho_{1} \cdot w_{1}$, finally we obtain that

$$
\left.U_{1}\left(x_{1}, x_{3}\right)\right|_{x_{3}=0}=\vartheta_{i} \cdot \rho_{\text {Land }} \cdot \frac{d X_{3}\left(x_{1}\right)}{d x_{1}}
$$

In order to obtain the corresponding conditions on the surface $\partial S$, at first, let's write the equation of surface of the section as

$$
x_{3}=h+X_{3, \partial S}\left(x_{1}\right),
$$

where $X_{3, \partial S}\left(x_{1}\right)$ is a perturbation of surface of the section $\partial S$ (it is also assumed to be an infinitesimal: refer to (14)).

Then we will obtain

$$
\left.\left[p_{1}+\tilde{p}_{1}\right]\right|_{x_{3}=h+X_{3,2 s}}=\left.\left[p_{2}+\tilde{p}_{2}\right]\right|_{x_{3}=h+X_{3,2 s}} .
$$

From expansion

$$
\left.p_{i}\right|_{x_{3}=h+X_{3, \partial S}}=\left.p_{i}\right|_{x_{3}=h}+\left.\frac{\partial p_{i}}{\partial x_{3}}\right|_{x_{3}=h} \cdot X_{3, \partial S}+o\left(X_{3, \partial S}^{2}\right) \quad(i=1,2),
$$

if taking into account that $\left.p_{1}\right|_{x_{3}=h-0}=\left.p_{2}\right|_{x_{3}=h+0}$ (i.e. equality of pressures on both sides of the section surface) and $\frac{\partial p_{i}}{\partial x_{3}}+g \cdot \rho_{i}=0 \quad(i=1,2)$ (i.e. the equation (6)), then after neglecting of infinitesimals higher than the first order we obtain

$$
\left.\tilde{p}_{1}\right|_{x_{3}=h}-\left.\tilde{p}_{2}\right|_{x_{3}=h}=g \cdot\left(\left.\tilde{\rho}_{1}\right|_{x_{3}=h}-\left.\tilde{\rho}_{2}\right|_{x_{3}=h}\right) \cdot X_{3, \partial S}\left(x_{1}\right) .
$$

Therefore, taking into account equalities of the section surface normal component velocities (analogical to the condition on the Earth surface), we obtain

$$
\left.\vartheta_{1}\left(x_{3}\right)\right|_{x_{3}=h} \cdot \frac{d X_{3, \partial S}\left(x_{1}\right)}{d x_{1}}-\left.w_{1}\left(x_{1}, x_{3}\right)\right|_{x_{3}=h}=\left.\vartheta_{2}\left(x_{3}\right)\right|_{x_{3}=h} \cdot \frac{d X_{3, \partial S}\left(x_{1}\right)}{d x_{1}}-\left.w_{2}\left(x_{1}, x_{3}\right)\right|_{x_{3}=h}=0 .
$$

From this

$$
\frac{d X_{3, \partial S}\left(x_{1}\right)}{d x_{1}}=\frac{w_{1}\left(x_{1}, h\right)-w_{2}\left(x_{1}, h\right)}{\vartheta_{1}(h)-\vartheta_{2}(h)},
$$




$$
w_{1}\left(x_{1}, h\right) \cdot \vartheta_{1}(h)=w_{2}\left(x_{1}, h\right) \cdot \vartheta_{2}(h) .
$$

In (20), taking into account notation $U_{i}=\rho_{i} \cdot w_{i}, \quad i=1,2$, we obtain

$$
\left.\left\{U_{1} \cdot \vartheta_{2} \cdot \rho_{2}\right\}\right|_{x_{3}=h}=\left.\left\{U_{2} \cdot \vartheta_{1} \cdot \rho_{1}\right\}\right|_{x_{3}=h} .
$$

Therefore, conditions (16), (18), (19) and (21) are the necessary conditions of consistency on the section surface $\partial S$ of two mediums $S 1$ and $S 2$ and on the Earth surface.

Now we will require that functions introduced in (14) would satisfy equations (12) of the perturbed air flow and conditions (16), (18), (19), (21). Substituting functions form (14) in turn into the corresponding equations of the system (12) taking into account consistency conditions (16), (18), (19), (21), we obtain the following system:

$$
\begin{aligned}
& \gamma \cdot \vartheta_{i}\left(x_{3}\right) \cdot U_{i}^{(1)}\left(x_{3}\right)+\left\{\tilde{p}_{i}^{(1)}\left(x_{3}\right)\right\}^{\prime}+g \cdot \tilde{\rho}_{i}^{(1)}\left(x_{3}\right)=0, \\
& \vartheta_{i}\left(x_{3}\right) \cdot \tilde{V}_{i}^{(1)}\left(x_{3}\right)+\tilde{p}_{i}^{(1)}\left(x_{3}\right)=0, \\
& \gamma \cdot \vartheta_{i}\left(x_{3}\right) \cdot \tilde{\rho}_{i}^{(1)}\left(x_{3}\right)+\gamma \cdot \tilde{V}_{i}^{(1)}\left(x_{3}\right)-\left\{U_{i}^{(1)}\left(x_{3}\right)\right\}^{\prime}=0, \\
& \gamma \cdot \vartheta_{i}\left(x_{3}\right) \cdot \tilde{p}_{i}^{(1)}\left(x_{3}\right)-\gamma \cdot \bar{T}_{i}\left(x_{3}\right) \cdot \vartheta_{i}\left(x_{3}\right) \cdot \tilde{\rho}_{i}^{(1)}\left(x_{3}\right)-d_{i} \cdot U_{i}^{(1)}\left(x_{3}\right)=0, \\
& U_{1}^{(1)}(0)+\vartheta_{1}(0) \cdot \rho_{\text {Land }} \cdot C \cdot \gamma=0, \\
& \tilde{p}_{1}^{(1)}(h)+\tilde{p}_{2}^{(1)}(h)=C \cdot g \cdot\left(\rho_{1}(h)-\rho_{2}(h)\right), \\
& C \cdot \gamma \cdot\left(\vartheta_{1}(h)-\vartheta_{2}(h)\right) \cdot \rho_{1}(h) \cdot \rho_{2}(h)=\rho_{2}(h) \cdot U_{1}^{(1)}(h)-\rho_{1}(h) \cdot U_{2}^{(1)}(h), \\
& \rho_{2}(h) \cdot \vartheta_{2}(h) \cdot U_{1}^{(1)}(h)=\rho_{1}(h) \cdot \vartheta_{1}(h) \cdot U_{2}^{(1)}(h) .
\end{aligned}
$$

Remark 1. Boundary conditions (28)-(29) on the surface of the section $\partial S$ give interesting equalities

$$
\left.\left[U_{i}^{(1)}\left(x_{3}\right)+C \cdot \gamma \cdot \rho_{i}\left(x_{3}\right) \cdot \vartheta_{i}\left(x_{3}\right)\right]\right|_{x_{3}=h}=0 \quad(i=1,2) .
$$

Remark 2. System of equations (22)-(25) with boundary conditions (26)-(29) is a complete formulation of the problem for finding asymptotic solutions of the system of equations (12) of the perturbed airflow. However, it is easy to prove that the boundary conditions (26)-(29) are not sufficient for a unique solution of the system (22)-(25). Additional conditions, which allow to find the solution of the system (22)-(25) unique, with boundary conditions (26)-(29) (system (12) with boundary conditions (16), (18), (19), (21)), will be formulated further on. Meanwhile, we will just speak about solution of the system (22)-(29) (systems (12), (16), (18), (19), (21)), just implying that the corresponding missing conditions exist for the unique solution thereof.

Now let us do proceed this way:

- from (23) we have that 


$$
\tilde{p}_{i}^{(1)}\left(x_{3}\right)=-\vartheta_{i}\left(x_{3}\right) \cdot \tilde{V}_{i}^{(1)}\left(x_{3}\right)
$$

- form (25) and (30) we have that

$$
\gamma \cdot \vartheta_{i}^{2}\left(x_{3}\right) \cdot \tilde{V}_{i}^{(1)}\left(x_{3}\right)-\gamma \cdot \bar{T}_{i}\left(x_{3}\right) \cdot \vartheta_{i}\left(x_{3}\right) \cdot \tilde{\rho}_{i}^{(1)}\left(x_{3}\right)-d_{i} \cdot U_{i}^{(1)}\left(x_{3}\right)=0
$$

- from (24) we have that

$$
\tilde{\rho}_{i}^{(1)}\left(x_{3}\right)=\frac{\left\{U_{i}^{(1)}\left(x_{3}\right)\right\}^{\prime}-\gamma \cdot \tilde{V}_{i}^{(1)}\left(x_{3}\right)}{\gamma \cdot \vartheta_{i}\left(x_{3}\right)}
$$

- from (31) and (32) we have that

$$
\tilde{V}_{i}^{(1)}\left(x_{3}\right)=\frac{\bar{T}_{i}\left(x_{3}\right) \cdot\left\{U_{i}^{(1)}\left(x_{3}\right)\right\}^{\prime}+d_{i} \cdot U_{i}^{(1)}\left(x_{3}\right)}{\gamma \cdot\left\{\bar{T}_{i}\left(x_{3}\right)-\vartheta_{i}^{2}\left(x_{3}\right)\right\}} ;
$$

- from (30) and (33) we obtain that

$$
\tilde{p}_{i}^{(1)}\left(x_{3}\right)=-\frac{\vartheta_{i}\left(x_{3}\right)}{\gamma} \cdot \frac{\bar{T}_{i}\left(x_{3}\right) \cdot\left\{U_{i}^{(1)}\left(x_{3}\right)\right\}^{\prime}+d_{i} \cdot U_{i}^{(1)}\left(x_{3}\right)}{\bar{T}_{i}\left(x_{3}\right)-\vartheta_{i}^{2}\left(x_{3}\right)} ;
$$

- from (32) and (35) we obtain that

$$
\tilde{\rho}_{i}^{(1)}\left(x_{3}\right)=-\frac{1}{\gamma \cdot \vartheta_{i}\left(x_{3}\right)} \cdot \frac{\vartheta_{i}^{2}\left(x_{3}\right) \cdot\left\{U_{i}^{(1)}\left(x_{3}\right)\right\}^{\prime}+d_{i} \cdot U_{i}^{(1)}\left(x_{3}\right)}{\bar{T}_{i}\left(x_{3}\right)-\vartheta_{i}^{2}\left(x_{3}\right)} .
$$

Now then, the desired functions $\tilde{V}_{i}^{(1)}\left(x_{3}\right), \tilde{p}_{i}^{(1)}\left(x_{3}\right)$ and $\tilde{\rho}_{i}^{(1)}\left(x_{3}\right)(i=1,2)$, as may be inferred from (33)-(35), are expressed in terms of functions $U_{i}^{(1)}\left(x_{3}\right)$ and $\left\{U_{i}^{(1)}\left(x_{3}\right)\right\}^{\prime}$. Taking into account these three expressions in the last "untouched" equation (22), we obtain that

$$
\begin{aligned}
& \gamma^{2} \cdot \vartheta_{i}^{2}\left(x_{3}\right) \cdot\left(\bar{T}_{i}\left(x_{3}\right)-\vartheta_{i}^{2}\left(x_{3}\right)\right)^{2} \cdot U_{i}^{(1)}\left(x_{3}\right)-g \cdot \vartheta_{i}^{2}\left(x_{3}\right) \cdot\left(\bar{T}_{i}\left(x_{3}\right)-\vartheta_{i}^{2}\left(x_{3}\right)\right) \cdot\left\{U_{i}^{(1)}\left(x_{3}\right)\right\}^{\prime} \\
& -g \cdot d_{i} \cdot\left(\bar{T}_{i}\left(x_{3}\right)-\vartheta_{i}^{2}\left(x_{3}\right)\right) \cdot U_{i}^{(1)}\left(x_{3}\right) \\
& -\bar{T}_{i}\left(x_{3}\right) \cdot \vartheta_{i}\left(x_{3}\right) \cdot \vartheta_{i}^{\prime}\left(x_{3}\right) \cdot\left(\bar{T}_{i}\left(x_{3}\right)-\vartheta_{i}^{2}\left(x_{3}\right)\right) \cdot\left\{U_{i}^{(1)}\left(x_{3}\right)\right\}^{\prime} \\
& -d_{i} \cdot \vartheta_{i}\left(x_{3}\right) \cdot \vartheta_{i}^{\prime}\left(x_{3}\right) \cdot\left(\bar{T}_{i}\left(x_{3}\right)-\vartheta_{i}^{2}\left(x_{3}\right)\right) \cdot U_{i}^{(1)}\left(x_{3}\right) \\
& -\bar{T}_{i}^{\prime}\left(x_{3}\right) \cdot \vartheta_{i}^{2}\left(x_{3}\right) \cdot\left(\bar{T}_{i}\left(x_{3}\right)-\vartheta_{i}^{2}\left(x_{3}\right)\right)\left\{U_{i}^{(1)}\left(x_{3}\right)\right\}^{\prime} \\
& -\bar{T}_{i}\left(x_{3}\right) \cdot \vartheta_{i}^{2}\left(x_{3}\right) \cdot\left(\bar{T}_{i}\left(x_{3}\right)-\vartheta_{i}^{2}\left(x_{3}\right)\right) \cdot\left\{U_{i}^{(1)}\left(x_{3}\right)\right\}^{\prime \prime} \\
& -d_{i} \cdot \vartheta_{i}^{2}\left(x_{3}\right) \cdot\left(\bar{T}_{i}\left(x_{3}\right)-\vartheta_{i}^{2}\left(x_{3}\right)\right) \cdot\left\{U_{i}^{(1)}\left(x_{3}\right)\right\}^{\prime}
\end{aligned}
$$




$$
\begin{aligned}
& +\bar{T}_{i}\left(x_{3}\right) \cdot \bar{T}_{i}^{\prime}\left(x_{3}\right) \cdot \vartheta_{i}^{2}\left(x_{3}\right) \cdot\left\{U_{i}^{(1)}\left(x_{3}\right)\right\}^{\prime}-2 \cdot \bar{T}_{i}\left(x_{3}\right) \cdot \vartheta_{i}^{3}\left(x_{3}\right) \cdot \vartheta_{i}^{\prime}\left(x_{3}\right)\left\{U_{i}^{(1)}\left(x_{3}\right)\right\}^{\prime} \\
& +d_{i} \cdot \bar{T}_{i}^{\prime}\left(x_{3}\right) \cdot \vartheta_{i}^{2}\left(x_{3}\right) \cdot U_{i}^{(1)}\left(x_{3}\right)-2 \cdot d_{i} \cdot \vartheta_{i}^{3}\left(x_{3}\right) \cdot \vartheta_{i}^{\prime}\left(x_{3}\right) \cdot U_{i}^{(1)}\left(x_{3}\right)=0 .
\end{aligned}
$$

In the last equation let's take into account that $\bar{T}_{i}^{\prime}\left(x_{3}\right) \equiv-\alpha_{i} \quad(i=1,2)$, and then let's group the corresponding coefficients at $U_{i}^{(1)}\left(x_{3}\right),\left\{U_{i}^{(1)}\left(x_{3}\right)\right\}^{\prime}$ and $\left\{U_{i}^{(1)}\left(x_{3}\right)\right\}^{\prime \prime}$. Then we will obtain the following second order differential equation in relation to the desired function $U_{i}^{(1)}\left(x_{3}\right)$ :

$$
\begin{aligned}
& \bar{T}_{i}\left(x_{3}\right) \cdot \vartheta_{i}^{2}\left(x_{3}\right) \cdot\left(\bar{T}_{i}\left(x_{3}\right)-\vartheta_{i}^{2}\left(x_{3}\right)\right) \cdot\left\{U_{i}^{(1)}\left(x_{3}\right)\right\}^{\prime \prime} \\
& +\left\{g \cdot \beta \cdot \bar{T}_{i}\left(x_{3}\right) \cdot \vartheta_{i}^{2}\left(x_{3}\right)+2 \cdot \beta \cdot R \cdot \alpha_{i} \cdot \vartheta_{i}^{4}\left(x_{3}\right)-g \cdot \beta \cdot \vartheta_{i}^{4}\left(x_{3}\right)-\alpha_{i} \cdot \beta \cdot R \cdot \bar{T}_{i}\left(x_{3}\right) \cdot \vartheta_{i}^{2}\left(x_{3}\right)\right\} \cdot\left\{U_{i}^{(1)}\left(x_{3}\right)\right\} \\
& +\left\{g \cdot d_{i} \cdot\left(\bar{T}_{i}\left(x_{3}\right)-\vartheta_{i}^{2}\left(x_{3}\right)\right)-\beta^{2} \cdot \vartheta_{i}^{2}\left(x_{3}\right) \cdot\left(\bar{T}_{i}\left(x_{3}\right)-\vartheta_{i}^{2}\left(x_{3}\right)\right)\right. \\
& \left.+\beta \cdot R \cdot \alpha_{i} \cdot d_{i} \cdot \vartheta_{i}^{2}\left(x_{3}\right)\right\} \cdot U_{i}^{(1)}\left(x_{3}\right)=0 .
\end{aligned}
$$

Let us study the equation (36). It is to notice that the coefficient at the highest derivative $\left\{U_{i}^{(1)}\left(x_{3}\right)\right\}^{\prime \prime}$ will be equal to zero only at two points, namely, at the points, where $\bar{T}_{i}\left(x_{3}\right) \cdot \vartheta_{i}^{2}\left(x_{3}\right)=0$ and $\bar{T}_{i}\left(x_{3}\right)-\vartheta_{i}^{2}\left(x_{3}\right)=0$.

In other words, the equation (36) is the equation with two regular critical points. To the first critical point corresponds (since $\vartheta_{i}\left(x_{3}\right) \not \equiv 0 \forall x_{3} \quad(i=1,2)$,) a point, at which $T_{i}\left(x_{3}\right)=0$.

Since $T_{\text {Land }} \not \equiv 0$ at $T_{1}\left(x_{3}\right) \stackrel{\text { def }}{\equiv} T_{\text {Land }}+\alpha_{1} \cdot x_{3}$, we obtain that for the second layer $S 2$ the given critical point is at the upper border thereof and therefore cannot be inside the first medium $S 1$.

The second critical point, for which $\vartheta_{i}^{2}\left(x_{3}\right)=\bar{T}_{i}\left(x_{3}\right) \equiv \beta \cdot R \cdot T_{i}\left(x_{3}\right) \quad(i=1,2)$, can be inside the first layer as well the second one. Therefore, at this critical point we have to require for finiteness of functions $U_{i}^{(1)}\left(x_{3}\right), \tilde{V}_{i}^{(1)}\left(x_{3}\right), \tilde{p}_{i}^{(1)}\left(x_{3}\right)$ and $\tilde{\rho}_{i}^{(1)}\left(x_{3}\right)$. In order to study the second critical point (i.e. the point, which can be interpreted as the point, at which the velocity of the flow $\vartheta_{i}\left(x_{3}\right)$ is equal to the sound velocity $\beta \cdot R \cdot T_{i}\left(x_{3}\right)$ ) and to formulate the conditions determining the finiteness of functions $U_{i}^{(1)}\left(x_{3}\right), \tilde{V}_{i}^{(1)}\left(x_{3}\right), \tilde{p}_{i}^{(1)}\left(x_{3}\right)$ and $\tilde{\rho}_{i}^{(1)}\left(x_{3}\right)$ at the second critical point, let's introduce a new independent variable $\varphi$, which is determined by equality

$$
\varphi \equiv \frac{\bar{T}_{i}\left(x_{3}\right)-\vartheta_{i}^{2}\left(x_{3}\right)}{\vartheta_{i}^{2}\left(x_{3}\right)} .
$$

Remark 3. Finding the conditions, satisfaction of which provides the finiteness of functions $U_{i}^{(1)}\left(x_{3}\right), \tilde{V}_{i}^{(1)}\left(x_{3}\right), \tilde{p}_{i}^{(1)}\left(x_{3}\right)$ and $\tilde{\rho}_{i}^{(1)}\left(x_{3}\right)$ (and so, functions from (14)), is the provision of unique 
solubility of the system of equations (22)-(25) with boundary conditions (26)-(29). In other words, the conditions, which provide the finiteness of functions $U_{i}^{(1)}\left(x_{3}\right), \tilde{V}_{i}^{(1)}\left(x_{3}\right), \tilde{p}_{i}^{(1)}\left(x_{3}\right)$ and $\tilde{\rho}_{i}^{(1)}\left(x_{3}\right)$ at the second critical point of the equation (36), will be those missing additional conditions (see Remark 2), which together with boundary conditions (26)-(29) provide the proof of unique solubility of the system (22)-(25).

Remark 4. From formulae (33)-(35) it follows that it is sufficient to formulate a condition of finiteness only for function $U_{i}^{(1)}\left(x_{3}\right)$ at the second critical point of the equation (36), namely,

- with the help of the formula (33) the corresponding condition for finiteness of the function $\tilde{V}_{i}^{(1)}\left(x_{3}\right)$ at the second critical point of the equation (36) will "automatically" be formulated;

- with the help of the formula (34) - the corresponding condition for the function $\tilde{p}_{i}^{(1)}\left(x_{3}\right)$;

- with the help of the formula (35) - the corresponding condition for the function $\tilde{\rho}_{i}^{(1)}\left(x_{3}\right)$.

Taking into account the new independent variable $\varphi$, determined by the (37), the equation (36) will look more simple:

$$
\begin{aligned}
& \varphi \cdot(\varphi+1) \cdot\left\{U_{i}^{(1)}(\varphi)\right\}_{\varphi}^{\prime \prime}+\frac{\alpha_{i} \cdot R \cdot(\varphi-1)-g \cdot \varphi}{\alpha_{i} \cdot R} \cdot\left\{U_{i}^{(1)}(\varphi)\right\}_{\varphi}^{\prime} \\
& +\frac{\beta \cdot\left(g-\alpha_{i} \cdot R\right) \cdot\left(\beta \cdot R \cdot \alpha_{i}+g \cdot \varphi\right)-g \cdot\left(\beta \cdot R \cdot \alpha_{i}+g \cdot \varphi\right)-\gamma^{2} \cdot \vartheta_{i}^{4}(\varphi) \cdot \varphi^{2}}{\beta^{2} \cdot R^{2} \cdot \alpha_{i}^{2}} \cdot U_{i}^{(1)}(\varphi)=0 .
\end{aligned}
$$

It is obvious that for the equation (38) the regular critical point will be the point $\varphi=0$ (it can be seen in the transformation (38) (see [8-9]). For this let's multiply the equation (38) by $\varphi$ and divide by $\varphi+1$ :

$$
\varphi^{2} \cdot\left\{U_{i}^{(1)}(\varphi)\right\}_{\varphi}^{\prime \prime}+\varphi \cdot F_{1}(\varphi) \cdot\left\{U_{i}^{(1)}(\varphi)\right\}_{\varphi}^{\prime}+F_{2}(\varphi) \cdot U_{i}^{(1)}(\varphi)=0
$$

where

$$
\begin{aligned}
& F_{1}(\varphi) \stackrel{\text { def }}{\equiv} \frac{\alpha_{i} \cdot R \cdot(\varphi-1)-g \cdot \varphi}{\alpha_{i} \cdot R \cdot(\varphi+1)} \\
& F_{2}(\varphi) \stackrel{\text { def }}{\equiv} \frac{\beta \cdot\left(g-\alpha_{i} \cdot R\right) \cdot\left(\beta \cdot R \cdot \alpha_{i}+g \cdot \varphi\right)-g \cdot\left(\beta \cdot R \cdot \alpha_{i}+g \cdot \varphi\right)-\gamma^{2} \cdot \vartheta_{i}^{4}(\varphi) \cdot \varphi^{2}}{\beta^{2} \cdot R^{2} \cdot \alpha_{i}^{2} \cdot(\varphi+1)} .
\end{aligned}
$$

Setting the functions $F_{1}(\varphi)$ and $F_{2}(\varphi)$ as

$$
\left\{\begin{array}{l}
F_{1}(\varphi)=\sum_{n=0}^{\infty} f_{n}^{(1)} \cdot \varphi^{n}, \\
F_{2}(\varphi)=\sum_{n=0}^{\infty} f_{n}^{(2)} \cdot \varphi^{n},
\end{array}\right.
$$


where $f_{n}^{(1)}$ and $f_{n}^{(2)}(n=0,1,2, \ldots)$ are constant and the series in (40) converge in $D \stackrel{\text { def }}{\equiv}\{\varphi:|\varphi| \leq r\}$, i.e. in the closed circle with its centre at the critical point $\varphi=0$ and radius $r$, then, depicting the desired solution as $U_{i}^{(1)}(\varphi)$ of the equation (39) in $D$ (more precisely, the holomorphic solution at the point $\varphi=0)$

$$
U_{i}^{(1)}(\varphi)=\varphi^{\lambda} \cdot\left[1+\sum_{n=1}^{\infty} U_{n} \cdot \varphi^{n}\right]
$$

where $U_{n}(n=1,2, \ldots)$ are yet unknown constants, $\lambda$ is yet unknown number, from (39)-(41) we get that

$$
\begin{aligned}
& \varphi^{\lambda} \cdot\left[\lambda \cdot(\lambda+1)+\sum_{n=1}^{\infty} U_{n} \cdot(\lambda+n) \cdot(\lambda+n-1) \cdot \varphi^{n}\right]+\varphi^{\lambda} \cdot\left[\lambda+\sum_{n=1}^{\infty} U_{n} \cdot(\lambda+n) \cdot \varphi^{n}\right] \cdot \sum_{n=0}^{\infty} f_{n}^{(1)} \cdot \varphi^{n} \\
& +\varphi^{\lambda} \cdot\left[1+\sum_{n=1}^{\infty} U_{n} \cdot \varphi^{n}\right] \cdot \sum_{n=0}^{\infty} f_{n}^{(2)} \cdot \varphi^{n}=0 .
\end{aligned}
$$

In the equation (42), multiplying the series and requiring that the left part of the obtained equation has to be identically equal to zero, we obtain the following system of algebraic equations in relation to the $\lambda$ and coefficients $U_{n}(n=1,2, \ldots)$ :

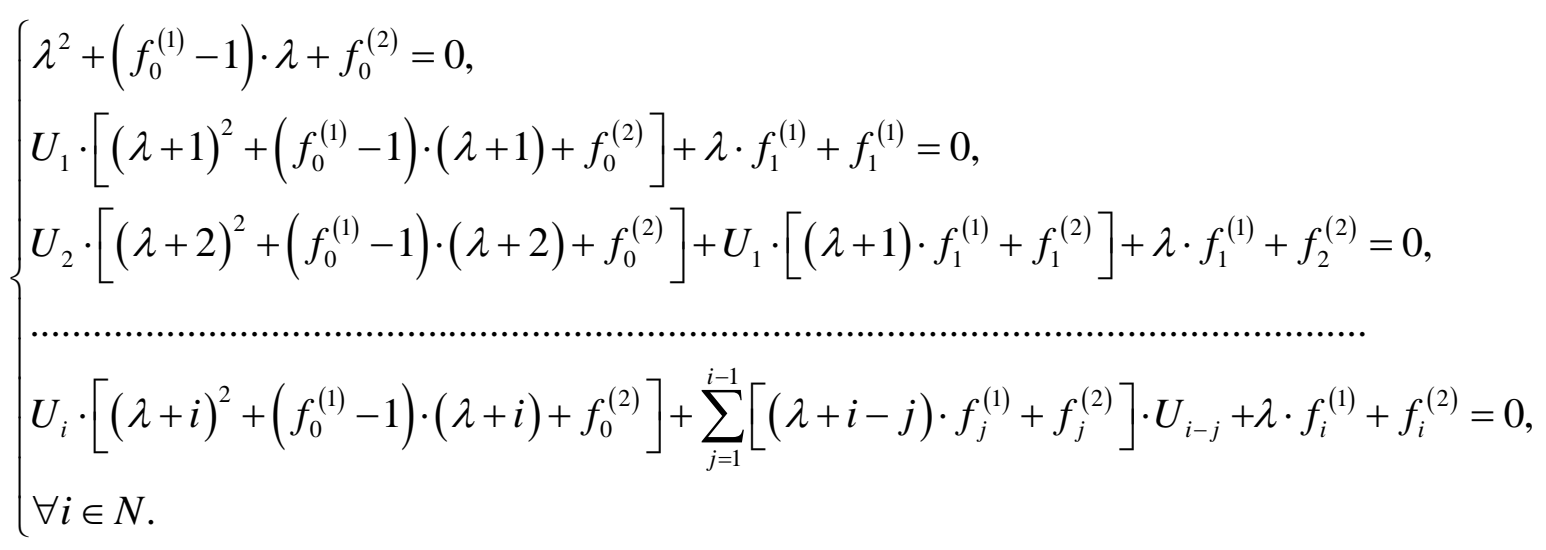

The first equation in the system (43) is a determining equation for (38) at the point $\varphi=0$. As it is known $([8,10])$, the roots of this equation are characteristic orders of the equation (38) in relation to the critical point $\varphi=0$. Since in equation (39) the function $F_{1}(\varphi)$, depicted according to (40) takes the form $F_{1}(\varphi)=-1+\left(1-\frac{g}{\alpha_{i} \cdot R}\right) \cdot \varphi$, then, obviously, $f_{0}^{(1)}=-1$. Analogously, for the function $F_{2}(\varphi)$, setting according to $(4)$, the first summand $f_{0}^{(2)}$ is equal to zero, i.e. $f_{0}^{(2)}=0$. Therefore, the determining equation for (38) at the critical point $\varphi=0$ takes the form $\lambda^{2}-2 \cdot \lambda=0$, the roots are $\lambda_{1}=0$ and $\lambda_{2}=2$. 
So, the characteristic orders of the equation (38) are $\lambda_{1}=0$ and $\lambda_{2}=2$. It is possible to verify easy and at once that the solution of equation (38), which corresponds to a characteristic order $\lambda_{1}=0$, has a logarithmic singularity (see [8-10]). If we return to notation with variable $x_{3}$ (see Eq. 37), then, according to the formula (34) we obtain that at the point $\varphi=0$ the pressure $\tilde{p}_{i}^{(1)}\left(x_{3}\right)$ becomes infinite. Therefore, it is necessary to take only such solution of equation (38), which corresponds to the characteristic order $\lambda_{2}=2$. The solution, corresponding to this characteristic order, is in effect in the second layer $S 2$ and it turns the function $U_{i}^{(1)}\left(x_{3}\right)$ to zero, what means the "vertical velocity" $w_{2}\left(x_{1}, x_{3}\right)$ turns to zero. It can be interpreted as a presence of a "sturdy wall" in the form of surface, on which there is a critical point $\varphi=0$, i.e. on which

$$
0=\varphi=\frac{\bar{T}_{2}\left(x_{3}\right)-\vartheta_{2}^{2}\left(x_{3}\right)}{\vartheta_{2}^{2}\left(x_{3}\right)} .
$$

This means $\vartheta_{2}^{2}\left(x_{3}\right)=\bar{T}_{2}\left(x_{3}\right)$. In other words, the critical point $x_{3} \in S 2$, in which $\vartheta_{2}^{2}\left(x_{3}\right)=\bar{T}_{2}\left(x_{3}\right)$, defines a "sturdy wall"-surface, described by the equality $\vartheta_{2}^{2}\left(x_{3}\right)=\beta \cdot R \cdot T_{2}\left(x_{3}\right)$.

Now, let us return to the system (43). Let us notice that in all equations of this system, except the first one, coefficients at $U_{n}(n=1,2, \ldots)$ are equal to $(\lambda+n)^{2}+\left(f_{0}^{(1)}-1\right) \cdot(\lambda+n)+f_{0}^{(2)}$, i.e., if the left part of the first equation is set as $\aleph(\lambda) \stackrel{\text { def }}{\equiv} \lambda^{2}+\left(f_{0}^{(1)}-1\right) \cdot \lambda+f_{0}^{(2)}$, then coefficients at $U_{n}(n=1,2, \ldots)$ are equal to $\aleph(\lambda+n)(n=1,2, \ldots)$.

In addition, if we assume that

$$
\begin{aligned}
& \lambda=\lambda_{1} \Rightarrow \lambda_{2} \neq \lambda_{1}+n, \forall n \in \mathbb{N}, \\
& \lambda=\lambda_{2} \Rightarrow \lambda_{1} \neq \lambda_{2}+n, \forall n \in \mathbb{N},
\end{aligned}
$$

where $\lambda_{1}$ and $\lambda_{2}$ are the roots of the equation $\aleph(\lambda)=0$, then conditions $\aleph(\lambda+n) \neq 0$ will be fulfilled for each $n \in \mathbb{N}$, moreover, the condition $\aleph(\lambda+n) \neq 0$ for each $n=1,2, \ldots$ can be expressed by a recurrent formula. Therefore, if the equality of the characteristic measures are not integer, then for the equation

$$
U_{i} \cdot\left[(\lambda+i)^{2}+\left(f_{0}^{(1)}-1\right) \cdot(\lambda+i)+f_{0}^{(2)}\right]+\sum_{j=1}^{i-1}\left[(\lambda+i-j) \cdot f_{j}^{(1)}+f_{j}^{(2)}\right] \cdot U_{i-j}+\lambda \cdot f_{i}^{(1)}+f_{i}^{(2)}=0, i=1,2, \ldots
$$

can be recurrently found two sequences of coefficients $U_{i}$, giving two formal solutions of the equation (38). Since in our case the characteristic measures are integer numbers, it is necessary to build the second solution of the equation (38), which corresponds to this specific case. For this let 
us notice that the singularity of the point $\varphi=0$ was expressed with multipliers $\varphi^{\lambda_{1}}$ and $\varphi^{\lambda_{2}}$ in formula (41) for solutions and in (42) for the equation:

$$
\left\{\begin{array}{l}
\bar{U}_{i}^{(1)}(\varphi)=\varphi^{\lambda_{1}(=0)} \cdot\left[1+\sum_{n=1}^{\infty} U_{n} \cdot \varphi^{n}\right] \\
\overline{\bar{U}}_{i}^{(2)}(\varphi)=\varphi^{\lambda_{2}(=2)} \cdot\left[1+\sum_{n=1}^{\infty} U_{n} \cdot \varphi^{n}\right] .
\end{array}\right.
$$

It can be shown that these two solutions, corresponding to two characteristic measures $\lambda_{1}=0$ and $\lambda_{1}=2$, form a fundamental system of solutions of the equation (39) and therefore the equation (38).

Let us make such substitution on in the equation (39) $U_{i}(\varphi)=\bar{U}_{i}(\varphi) \cdot u(\varphi)$, where $\bar{U}_{i}(\varphi)$ is determined according to (44). Then we have got

$$
\varphi^{2} \cdot u^{\prime \prime}(\varphi)+\left[2 \cdot \varphi^{2} \cdot \frac{\left\{\bar{U}_{i}^{(1)}(\varphi)\right\}_{\varphi}^{\prime}}{\bar{U}_{i}^{(1)}(\varphi)}+\varphi \cdot F_{1}(\varphi)\right] \cdot u^{\prime}(\varphi)=0
$$

from which follows:

$$
\begin{aligned}
& \frac{u^{\prime \prime}(\varphi)}{u^{\prime}(\varphi)}=-2 \cdot \frac{\left\{\bar{U}_{i}^{(1)}(\varphi)\right\}^{\prime}}{\bar{U}_{i}^{(1)}(\varphi)}-\frac{F_{1}(\varphi)}{\varphi}, \\
& u^{\prime}(\varphi)=A \cdot \frac{1}{\left\{\bar{U}_{i}^{(1)}(\varphi)\right\}^{2}} \cdot e^{-\int \frac{F_{1}(\theta)}{\varphi} d \varphi}=A \cdot \frac{\varphi^{-2 \cdot \lambda_{1}-f_{0}^{(1)}}}{\left[1+\sum_{n=1}^{\infty} U_{n} \cdot \varphi^{n}\right]^{2}} \cdot e^{-\sum_{n=1}^{\infty} f_{n}^{(1)} \cdot \frac{\varphi^{n}}{n}}=A \cdot \frac{\varphi \cdot e^{-\sum_{n=1}^{\infty} f_{n}^{(1)} \cdot \frac{\varphi^{n}}{n}}}{\left[1+\sum_{n=1}^{\infty} U_{n} \cdot \varphi^{n}\right]^{2}}
\end{aligned}
$$

where $A$ is a constant of integration.

If we assume that

$$
\bar{u}(\varphi)=\frac{e^{-\sum_{n=1}^{\infty} f_{n}^{(1)} \cdot \frac{\varphi^{n}}{n}}}{\left[1+\sum_{n=1}^{\infty} U_{n} \cdot \varphi^{n}\right]^{2}}
$$

then $\left.\bar{u}(\varphi)\right|_{\varphi=0}=1$ and $\bar{u}(\varphi)$ is holomorphic in a circle with its centre at the critical point $\varphi=0$, which does not include other critical points, such as function $F_{1}(\varphi)$ and function $U_{i}^{(1)}(\varphi)=\varphi^{-\lambda_{1}} \cdot \bar{U}_{i}^{(1)}(\varphi)$

Inside this circle it is possible to write

$$
\bar{u}(\varphi)=1+\sum_{n=1}^{\infty} \bar{u}_{n} \cdot \varphi^{n}
$$

Since $\Delta \lambda \stackrel{\text { def }}{\equiv} \lambda_{2}-\lambda_{1}=+2$ and $-2 \cdot \lambda_{2}-f_{0}^{(1)}=-\Delta \lambda-1$, then from (45)-(46) it follows that 


$$
\bar{u}(\varphi)=B+A \cdot\left[-\frac{1}{\Delta \lambda} \cdot \varphi^{-\Delta \lambda}-\sum_{n=1}^{\Delta \lambda-1} \frac{\bar{u}_{n}}{\Delta \lambda-n} \cdot z^{n-\Delta \lambda}+\bar{u}_{\Delta \alpha} \cdot \ln \varphi+\sum_{n=\Delta \lambda+1}^{\infty} \frac{\bar{u}_{n}}{n-\Delta \lambda} \cdot \varphi^{n-\Delta \lambda}\right],
$$

where $B$ is also a certain constant of integration. In relation to (47) we remind one more time that $\Delta \lambda$ in our specific case, i.e. when characteristic measures of the equation (38) are integer numbers, is integer, namely $\Delta \lambda=2-0=2$. The formula (47) is developed for a more specific case, when $\Delta \lambda$ is a natural number. This formula looks like this for the specific case, when $\Delta \lambda=2$ :

$$
\bar{u}(\varphi)=B+A \cdot\left[-\frac{1}{2 \cdot \varphi^{2}}-\frac{\bar{u}_{1}}{\varphi}+\bar{u}_{2} \cdot \ln \varphi+\sum_{n=3}^{\infty} \frac{\bar{u}_{n}}{n-2} \cdot \varphi^{n-2}\right] .
$$

So, having (47) (or (48), taking into account that $\Delta \lambda=2$ ) and if $\varphi$ changes in a sufficiently small neighbourhood of the point $\varphi=0$, then the general solution of the equation (39) will look like

$$
B \cdot \bar{U}_{i}^{(1)}(\varphi)+A \cdot\left[\bar{u}_{\Delta \lambda} \cdot \bar{U}_{i}^{(1)}(\varphi) \cdot \ln \varphi+\overline{\bar{U}}_{i}^{(1)}(\varphi)\right]
$$

where

$$
\overline{\bar{U}}_{i}^{(1)}(\varphi) \stackrel{\text { def }}{\equiv} \varphi^{\lambda_{2}} \cdot\left[1+\sum_{n=1}^{\infty} U_{n} \cdot \varphi^{n}\right] \cdot \frac{\sum_{\substack{n=1 \\ n \neq \Delta \lambda}}^{\infty} \frac{\bar{u}_{n}}{n-\Delta \lambda} \cdot \varphi^{n}-\frac{1}{\Delta \lambda}}{\varphi^{\Delta \lambda}} .
$$

Formulae (49)-(50) demonstrate a general solution on the equation (39) for the case, when $\Delta \lambda \in \mathbb{N}$. Let us notice that in (49) there is a logarithmic function in an explicit form, which shows that there is a logarithmic singularity in the solution (it was mentioned above already). Substituting the formulae (44) and (49)-(50) in the boundary conditions (18)-(19), (21), we can find the constants of integrations можем определить константы $A$ and $B$, and thereby the functions $u^{\prime}(\varphi)$ and $\bar{u}(\varphi)$ will completely defined.

\section{Remark 5. If the roots of the determining equation}

$$
\aleph(\lambda) \stackrel{\text { def }}{\equiv} \lambda^{2}+\left(f_{0}^{(1)}-1\right) \cdot \lambda+f_{0}^{(2)}=0
$$

are the same, i.e. if $\lambda_{1}=\lambda_{2}$ (it means that $\Delta \lambda=0$ ), we will obtain

$$
\bar{u}(\varphi)=B+A \cdot\left[\ln \varphi+\sum_{n=1}^{\infty} \frac{\bar{u}_{n}}{n} \cdot \varphi^{n}\right],
$$

and, therefore, the general solution of the equation (39) will be

$$
B \cdot \bar{U}_{i}^{(1)}(\varphi)+A \cdot\left[\bar{U}_{i}^{(1)}(\varphi) \cdot \ln \varphi+\overline{\bar{U}}_{i}^{(1)}(\varphi)\right]
$$

where 


$$
\overline{\bar{U}}_{i}^{(1)}(\varphi) \stackrel{\text { def }}{\equiv} \varphi^{\lambda_{1}\left(=\lambda_{2}\right)} \cdot\left[1+\sum_{n=1}^{\infty} \overline{\bar{U}}_{n} \cdot \varphi^{n}\right]
$$

So, above was described a process/algorithm of finding a holomorphic solution of the equation (38) in a neighbourhood of the critical point $\varphi=0$. Throwing out the abovementioned analysis and passing to "the old" variable $x_{3}$ (see (37)), it is possible to rewrite this solution in an explicit form:

$$
U_{2}^{(1)}\left(x_{3}\right)=\frac{\left[\bar{T}_{2}\left(x_{3}\right)-\vartheta_{2}^{2}\left(x_{3}\right)\right]^{2}}{\vartheta_{2}^{4}\left(x_{3}\right)} \cdot \sum_{n=0}^{\infty} U_{n}\left(x_{3}\right) \cdot \frac{\left[\bar{T}_{2}\left(x_{3}\right)-\vartheta_{2}^{2}\left(x_{3}\right)\right]^{n}}{\vartheta_{2}^{2 \cdot n}\left(x_{3}\right)},
$$

where coefficients $U_{n}\left(x_{3}\right)$ are determined by a recurrent formula

$$
U_{n}\left(x_{3}\right)=\frac{\beta \cdot n \cdot[g-R \cdot(n+2)] \cdot U_{n-1}\left(x_{3}\right)-g \cdot d_{2} \cdot U_{n-2}\left(x_{3}\right)+\left\{\gamma^{2} \cdot \vartheta_{2}^{4}\left(x_{3}\right)\right\} \cdot U_{n-3}\left(x_{3}\right)}{\beta^{2} \cdot R^{2} \cdot \alpha_{2}^{2} \cdot n \cdot(n+2)} \quad(n=0,1, \ldots) .
$$

It is a reminder that the abovementioned calculations, including numbers and formulae (51)-(52), are obtained with a presumption (13), i.e. with a presumption that the shape of the studied Earth surface is given by an equation $x_{3}=X_{3}\left(x_{1}\right)=\cos \left(\gamma \cdot x_{1}\right)$ (see (13)), which is a particular case of the formulated general problem. Now, using the acquired results in relation to this particular case, we pass to the solution of the general problem. Assume that the shape of the Earth surface is given by an equation

$$
x_{3}=X_{3}\left(x_{1}\right)
$$

where it is required that $\int_{-\infty}^{+\infty}\left|X_{3}\left(x_{1}\right)\right| d x_{1}<+\infty$.

Then the general solution of the full problem can be written in the following form of Fourier integral:

$$
\begin{aligned}
& U_{i}^{(\text {General })}\left(x_{1}, x_{3}\right)=\frac{1}{\pi} \cdot \int_{0}^{+\infty} d \sigma_{1} \int_{-\infty}^{+\infty} U_{i}^{(1)}\left(\sigma_{1}, x_{3}\right) \cdot X_{3}\left(\sigma_{2}\right) \cdot \sin \left(\gamma \cdot\left(x_{1}-\sigma_{2}\right)\right) d \sigma_{2}, \\
& \tilde{V}_{i}^{(\text {General })}\left(x_{1}, x_{3}\right)=\frac{1}{\pi} \cdot \int_{0}^{+\infty} d \sigma_{1} \int_{-\infty}^{+\infty} \tilde{V}_{i}^{(1)}\left(\sigma_{1}, x_{3}\right) \cdot X_{3}\left(\sigma_{2}\right) \cdot \cos \left(\gamma \cdot\left(x_{1}-\sigma_{2}\right)\right) d \sigma_{2}, \\
& \tilde{\rho}_{i}^{(\text {General })}\left(x_{1}, x_{3}\right)=\frac{1}{\pi} \cdot \int_{0}^{+\infty} d \sigma_{1} \int_{-\infty}^{+\infty} \tilde{\rho}_{i}^{(1)}\left(\sigma_{1}, x_{3}\right) \cdot X_{3}\left(\sigma_{2}\right) \cdot \cos \left(\gamma \cdot\left(x_{1}-\sigma_{2}\right)\right) d \sigma_{2}, \\
& p_{i}^{(\text {General })}\left(x_{1}, x_{3}\right)=\frac{1}{\pi} \cdot \int_{0}^{+\infty} d \sigma_{1} \int_{-\infty}^{+\infty} p_{i}^{(1)}\left(\sigma_{1}, x_{3}\right) \cdot X_{3}\left(\sigma_{2}\right) \cdot \cos \left(\gamma \cdot\left(x_{1}-\sigma_{2}\right)\right) d \sigma_{2}, \\
& X_{3, \text { OS }}\left(x_{1}\right)=\frac{1}{\pi} \cdot \int_{0}^{+\infty} d \sigma_{1} \int_{-\infty}^{+\infty} X_{3, \partial S}\left(\sigma_{1}\right) \cdot Z\left(\sigma_{2}\right) \cdot \cos \left(\gamma \cdot\left(x_{1}-\sigma_{2}\right)\right) d \sigma_{2},
\end{aligned}
$$


where $\tilde{p}_{i}, \tilde{\rho}_{i}, U_{i}^{(1)}, \tilde{V}_{i}^{(1)}$ and $X_{3, O S}$ are solutions, which correspond to the considered case supposedly (13).

Remark 6. When solutions $\tilde{p}_{i}, \tilde{\rho}_{i}, U_{i}^{(1)}, \tilde{V}_{i}^{(1)}$ have right critical points (it was studied above) and poles (i.e. wrong critical points) on the real coordinate axis, then in formulae from (54) for solutions $U_{i}^{(\text {General })}\left(x_{1}, x_{3}\right), \tilde{V}_{i}^{(\text {General })}\left(x_{1}, x_{3}\right), \tilde{\rho}_{i}^{(\text {General })}\left(x_{1}, x_{3}\right), p_{i}^{(\text {General })}\left(x_{1}, x_{3}\right), X_{3, \partial S}\left(x_{1}\right)$ of the complete problem, the corresponding integrations are understood as the main Cauchy value (for instance, see [11], [12]).

\section{References}

[1] L.D.Landau, E.M.Lifshitz. Statistical hidromechanics. - Moscow, USSR: Science Publishing House, 1986, 735 p.

[2] N.E.Kochin. About influence of earth relief on waves on the interface between two liquid masses with different densities. - Works of the main geophysical observatory: Theoretical meteorology, Issue 1, 1967, pp. 19-30.

[3] B.I.Izvekov, N.E.Kochin. Dynamical Meteorology. Part 2. - Novosibirsk: Hydrometeorologic Publishing House, 1971, 280 p.

[4] H.Lamb. Hydrodynamics. - Cambridge, UK: Cambridge University Press, 1975, $\mathrm{Xv}+738 \mathrm{p}$.

[5] V.Ya.Rudak. Mathematical Models of Natural Phenomenon and Engineering Processed. Part I. - Novosibirsk, Russia: Novosibirsk State Technical University Press, 2003, 181 p.

[6] A.S.Monin, A.M.Yaglom. Static Hydromechanics. Vol. II. - St. Petersburg, Russia: Hydrometeor Publishing, 1996, 620 p.

[7] Yu.L.Klimontovich. Turbulent Motion and Structure of Chaos. - Moscow, Russia: URSS Publishing, 2007, 328 p.

[8] L.S.Pontryagin. Ordinary Differential Equations. - Moscow, Russia: FizMatLit Publishing, 2001, 331 p.

[9] L.Ya.Adrianova. Introduction to the Theory of Linear Systems of Differential Equations.St. Petersburg, Russia: St. Petersburg State University Press, 1992, 239 p.

[10] E.T.Whittaker, G.N.Watson. A Course of Modern Analysis. - Cambridge, UK: Cambridge University Press, 1996, 608 p.

[11] V.A.Il'in, E.G.Poznyak. Fundamentals of Mathematical Analysis. Volumes I. - Moscow, Russia: FizMatLit Publishing, 2005, 648 p.

[12] V.A.Il'in, E.G.Poznyak. Fundamentals of Mathematical Analysis. Volumes II. - Moscow, Russia: FizMatLit Publishing, 2002, 454 p. 